\title{
Vorbemerkungen zum Sachstandsbericht: Entstehung und Grundsätze
}





\title{
Vorbemerkungen zum Sachstandsbericht Klimawandel: Entstehung und Grundsätze
}

\author{
Warum ein Sachstandsbericht Klimawandel für Österreich?
}

Der Klimawandel ist eine der größten Herausforderungen für die Menschheit in diesem Jahrhundert. Das „Intergovernmental Panel on Climate Change“ (IPCC) bestätigt in seinem 2013/2014 publizierten fünften Sachstandsbericht („Fifth Assessment Report, AR5") nochmals, was aus der Fachliteratur schon seit Jahrzehnten bekannt ist: Der Klimawandel findet statt und wird hauptsächlich durch menschliche Aktivitäten verursacht. Die mittlere globale Temperatur ist seit 1880 um fast ein Grad Celsius angestiegen. Des weiteren schlussfolgert das IPCC, dass zukünftig unverminderte Emissionen einen Temperaturanstieg um drei bis fünf Grad Celsius bis zum Ende des Jahrhunderts verursachen würden. Das IPCC Szenario mit dem höchsten Emissionsminderungsziel ist auf einen globalen Temperaturanstieg von zwei Grad Celsius bis Ende des Jahrhunderts ausgelegt - dies würde für Österreich einen Temperaturanstieg von rund vier Grad Celsius bedeuten; der bisherige mittlere Temperaturanstieg in Österreich ist ebenso bereits ungefähr zweimal so hoch wie der des globalen Durchschnitts. Die mit diesem globalen Szenario verknüpfte zukünftige Entwicklung würde bereits einen gefährlichen Klimawandel auslösen; die Emissionspfade mit höheren Emissionen können katastrophale Folgen nach sich ziehen.

Obwohl die Wissenschaft seit vielen Jahren auf den ständig fortschreitenden Klimawandel und seine Auswirkungen hinweist und trotz darauf basierender internationaler und nationaler Absichterklärungen den Klimawandel einzudämmen, nehmen die Treibhausgasemissionen weltweit weiterhin zu. Auch in Österreich zählen Klimawandelanpassung und Klimaschutz nicht zu den obersten Prioritäten der Politik. In manchen Diskussionen entsteht sogar der Eindruck, dieses Problem hätte mit Österreich wenig zu tun.

Angesichts des Ausmaßes der möglichen negativen Auswirkungen des Klimawandels und der Notwendigkeit sofort zu handeln, um den Klimawandel zu bremsen und das Klima zu stabilisieren, entstand die Idee, ähnlich den auf globaler Ebene erstellten IPCC Sachstandsberichten eine nationale Beurteilung für Österreich durchzuführen, einen Austrian Assessment Report 2014 (AAR14). Das nun vorliegende Buch ist das Ergebnis dieser Bemühungen. Es stellt einen IPCC-ähnlichen Bericht für Österreich dar, der bestehendes Wissen zum Klimawandel in Österreich, seine Auswirkungen, sowie die Erfordernisse und Möglichkeiten der Minderung und Anpassung in drei Bänden zusammenfasst, um einen ersten, umfassenden und konsolidierten Überblick über den Stand des Wissens zum Klimawandel in Österreich zu geben.

Statt eine kleine Gruppe von AutorInnen mit dieser Aufgabe zu betrauen, wurden alle zum Thema Klimawandel in Österreich Forschenden zur Mitwirkung eingeladen; die Bereitschaft war sehr groß. Letztendlich haben rund 240 WissenschaftlerInnen von rund 50 Forschungseinrichtungen den Sachstandsbericht Klimawandel (AAR14) gemeinsam erstellt. Es konnte solcherart ein wesentlich vollständigeres, disziplinenübergreifendes Bild gezeichnet werden, als dies einer kleine(ere)n Gruppe möglich gewesen wäre. Durch Zusammenführen von mit verschiedenen Ansätzen und Methoden erarbeiteten Erkenntnissen werden darüber hinaus die Ergebnisse robuster, bzw. werden weniger robuste als solche erkannt und der nach wie vor bestehende Forschungsbedarf tritt klarer zum Vorschein. Der Bericht stellt daher Entscheidungsgrundlagen für Entscheidungstragende auf unterschiedlichen Ebenen und in verschiedenen Sektoren bereit, deren Verlässlichkeit von den Forschenden gemeinsam abgeschätzt und transparent gemacht wurde (siehe dazu im Detail im Abschnitt „Umgang mit Unsicherheiten“ im Folgenden).

Die gemeinsame Anstrengung setzt auch ein deutliches und sichtbares Lebenszeichen der rasch wachsenden österreichischen „Klimaforschungscommunity“, die durch die gemeinsame Arbeit an diesem Bericht zusätzlich zusammengewachsen ist und von bisher nicht gekannten Synergien profitieren konnte.

\section{Aufbau und Grundsätze des AAR14 im Vergleich zum IPCC AR5}

Es war von Anbeginn vorgesehen, Erstellungsprozess und Struktur des Austrian Assessment Reports 2014 (AAR14) nach dem Muster des IPCC auszurichten, da sich zum einen das auf internationaler Ebene über die Jahre entwickelte Verfahren bewährt hat, und zum anderen seit dem vorletzten IPCC Sachstandsbericht (AR4) zudem weitere wesentliche Verbesserungen hinsichtlich Transparenz und Qualitätssicherung eingeführt wurden. Dennoch gibt es einige Abweichungen. 
Ähnlich den IPCC-Sachstandsberichten liegt dem AAR14 das Prinzip zugrunde, entscheidungsrelevant zu sein, aber keinen empfehlenden Charakter zu haben. Dieses Prinzip kommt vor allem im 3. Band zum Tragen. Der Bericht enthält daher somit auch zahlreiche Vorschläge, welche Maßnahmen möglich oder notwendig wären, wenn bestimmte Ergebnisse oder Ziele erreicht werden sollen. Um Handeln auch im Sinne des Vorsorgeprinzips zu ermöglichen (welches das Auftreten irreparabler Schäden, etwa an der Gesundheit oder an Ökosystemen, durch geeignete Maßnahmen von vornherein auszuschließen sucht), wird getrachtet, die volle Bandbreite („best case“ bis „worst case“) möglicher Auswirkungen des Klimawandels darzustellen. Da der „best case“ sehr häufig lediglich im Fortbestand der derzeitigen Situation besteht, liegt das Augenmerk naturgemäß stärker auf „worst case“ Szenarien. Diese sind keineswegs als Prognosen zu verstehen, oft nicht einmal als sehr wahrscheinliche Entwicklungen, aber ihre Betrachtung ist erforderlich, um der Gesellschaft informierte Entscheidungen zu ermöglichen.

Die Hauptinformationsquellen für die IPCC Berichte sind nach Begutachtungsverfahren publizierte Artikel in internationalen wissenschaftlichen Zeitschriften. Wiewohl sogenannte "graue “ Literatur ${ }^{1}$ vom IPCC nicht grundsätzlich ausgeschlossen wird, kommt ihr doch auf der internationalen Ebene eine geringe Rolle zu. Im AAR14 wurde verstärkte auch auf graue Literatur zurückgegriffen, jedoch (wie beim IPCC auch) unter der Voraussetzung, dass sie zugänglich ist, oder zugänglich gemacht wurde. Das erschien auf nationaler Ebene in größerem Ausmaß notwendig, weil Untersuchungen zu lokalen und regionalen Fragen oft nicht in referierten Zeitschriften publiziert werden, zum Stand des Wissens über den Klimawandel in Österreich aber dennoch Wesentliches beitragen können. Als Nebeneffekt des AAR14 ist daher nicht nur eine Literaturdatenbank entstanden, die alle Literaturzitate des Berichtes enthält, sondern auch eine Sammlung grauer Literatur, die über das Climate Change Centre Austria (CCCA) auch weiterhin zugänglich sein wird.

Ebenso haben die AutorInnen das übersetzte IPCC Glossar (aus dem IPCC AR4) an die Bedürfnisse des AAR14 angepasst und teilweise wesentlich erweitert. Ein gutes Beispiel dafür ist der Themenkreis „Boden“ der im IPCC Bericht wesentlich weniger ausführlich behandelt wird und daher im Glossar ungenügend berücksichtigt war. Das APCC Glossar wird vom CCCA als lebendes Dokument weitergeführt und online verfügbar gemacht.

Der Volltext des Austrian Assessment Report 2014, AAR14, ist in 3 Bände gegliedert. Band 1 widmet sich den physikalischen Grundlagen des Klimawandels, Band 2 den Auswirkungen, und Band 3 der möglichen Minderung von Treibhausgasemissionen und der Anpassung an den Klimawandel. Jeder der drei Bände wurde von zwei bis vier Co-Chairs betreut. Ihre Aufgabe war es, sicherzustellen, dass die einzelnen Kapitel keine wesentlichen Überschneidungen, Lücken, Inkonsistenzen, etc. aufweisen. Gemeinsam bilden die Co-Chairs der drei Bände das „Austrian Panel on Climate Change“ (APCC).

Diese Gliederung des AAR14 weicht von jener des IPCC AR5 insofern ab, als sich die Anpassungsmaßnahmen dort im zweiten Band zusammen mit den Auswirkungen des Klimawandels finden, weil Anpassung vor allem in natürlichen Systemen oft schwer von den Auswirkungen zu trennen ist. Dies war dem APCC bewusst, doch schien diesem wichtiger, dass Anpassungs- und Minderungsmaßnahmen nicht völlig von einander losgelöst betrachtet werden, da dies zu fehlgeleiteten Maßnahmen führen kann.

Jeder der Bände ist in 5 bis 6 Kapitel gegliedert. Für jedes Kapitel des Berichtes wurden zwei bis drei thematisch komplementäre, koordinierende LeitautorInnen bestimmt und vom Panel bestätigt, die ihrerseits KollegInnen einluden, Teile des Kapitels zu schreiben (LeitautorInnen), oder einzelne Beiträge zu liefern (Beitragende AutorInnen). Die koordinierenden LeitautorInnen organisierten die Arbeit innerhalb ihres Kapitels mit allen jenen, die bereit waren, einen Beitrag zu leisten. Sie tragen die Verantwortung für Inhalt und Fertigstellung der einzelnen Kapitel. Diese Organisation bzw. Hierarchie von AutorInnen entspricht ebenso der IPCC Praxis.

Der erste und - nach Feedback und Review überarbeitete - zweite Entwurf jedes Kapitels wurden jeweils einem anonymen externen Begutachtungsprozess unterzogen, ähnlich dem Peer-Reviewprozess von wissenschaftlichen Zeitschriften. Alle Kommentare und - im Falle des zweiten Entwurfes - auch die Antworten der AutorInnen wurden dokumentiert. Ähnlich dem IPCC Prozess stellten namentlich bekannte Review EditorInnen angemessene Reaktionen der AutorInnen auf jeden einzelnen Kommentar sicher. Anders als beim IPCC Bericht wurde die Regierung bzw. die Politik in den Begutachtungsprozess nicht explizit eingebunden, sehr wohl jedoch auch deren Feedbacks als Stakeholder zweimal eingeholt (siehe dazu auch im Folgenden).

Die Synthese stellt eine Zusammenfassung des Volltextes dar. Für sie zeichnet ein Redaktionsteam verantwortlich, das auf Basis von Beiträgen der koordinierenden LeitautorInnen eine geschlossene Darstellung der Ergebnisse erstellt hat. 
Die kürzeste Fassung, die Zusammenfassung für Entscheidungstragende, ist ebenfalls von einem Redaktionsteam auf Basis der Kernaussagen der einzelnen Kapitel entworfen worden, anschließend in einem Arbeitstreffen und mehrfachen Iterationsschleifen im Kreis der koordinierenden LeitautorInnen und vielen LeitautorInnen überarbeitet worden und letztendlich wieder vom Redaktionsteam finalisiert worden. Während die Zusammenfassung für Entscheidungstragende des IPCC Satz für Satz zwischen Wissenschaft und Politik ausgehandelt wird und das letztendlich veröffentlichte Papier dadurch politische Mächtigkeit erlangt, wurden politische Akteure in den AAR14 nach ausführlichen Diskussionen im APCC nur über zwei Stakeholder Workshops und das Angebot die Entwürfe zu kommentieren, eingebunden. Dies erschien dem APCC für einen kleinen Staat mit vielfältigen Abhängigkeiten wissenschaftlicher Institutionen und mit geringer Tradition in der Interaktion Wissenschaft-Politik als angemessene Vorgangsweise. Die Einbindung der Politik unterscheidet sich daher wesentlich von jener im IPCC Prozess.

Analog dem IPCC Bericht wurde die umfangreiche inhaltliche Arbeit von den Forschenden bzw. deren Institutionen unentgeltlich geleistet.

\section{Erstellung des Sachstandsberichtes}

Der Sachstandsbericht Klimawandel (AAR14) ist das Kernprodukt des im Rahmen des Austrian Climate Research Program (ACRP) des Klima- und Energiefonds (KLIEN) geförderten Projektes „Austrian Panel on Climate Change Assessment Report“, das eine Laufzeit vom 01.7.2011 bis 31.10.2014 hat. Die Leitung des Projektes lag bei Nebojsa Nakicenovic (Technische Universität Wien); ein Organisationskomitee, bestehend aus Helga Kromp-Kolb (Universität für Bodenkultur, Wien), Nebojsa Nakicenovic (Technische Universität Wien), und Karl Steininger (Universität Graz) hat gemeinsam mit dem Leiter des Review Prozesses, Keywan Riahi (International Institute for Applied Systems Analysis, IIASA) das APCC Projekt gesteuert. Über das Projekt wurden koordinative Tätigkeiten und Sachleistungen finanziert, aber die umfangreiche inhaltliche Arbeit der Forscherinnen und Forscher und die koordinative Tätigkeit des Organisationskomitees wurden unentgeltlich geleistet. Lediglich den Co-Chairs und den Koordinierenden Leitautorinnen wurde ein kleiner, einheitlicher Betrag zur Finanzierung von Hilfsdiensten, wie etwa die Erstellung von Abbildungen und Tabellen, oder die Unterstützung bei der Koordination der AutorInnen zur Verfügung gestellt. Der Fonds zur Förderung der wissenschaftlichen Forschung (FWF) hat einen Beitrag zur Drucklegung des Berichtes geleistet.

Die Einladung zur Mitwirkung an dem Projekt erging sowohl vor Projekteinreichung als auch nach Projektgenehmigung von der Projektleitung (Organisationskomitee) an die einschlägig Forschenden in Österreich, und nochmals von den Koordinierenden Leitautorinnen an die im jeweiligen Themenbereich Forschenden.

Potentielle künftige NutzerInnen des AAR14 aus Politik, Verwaltung, Zivilgesellschaft und Wirtschaft wurden in den Entstehungsprozess des AAR14 eingebunden, um deren Bedürfnissen möglichst gerecht zu werden. Die Stakeholder wurden zunächst zur vorgesehenen Struktur und Inhalt des Berichtes gefragt, und wieder als die vorläufigen Ergebnisse vorlagen (zweiter Entwurf). In beiden Fällen wurden jeweils Stakeholder-Konferenzen abgehalten und die Vorschläge und Kommentare der NutzerInnen anschließend durch die AutorInnen geprüft und soweit möglich und als innerhalb des APCC Rahmens als umsetzbar erachtet, in der weiteren Ausarbeitung berücksichtigt.

Ein Scientific Advisory Board (SAB), bestehend aus internationalen ExpertInnen, wurde zur Begleitung des Projektes eingerichtet.

Im Laufe der Arbeit hat sich allerdings gezeigt, dass beträchtliche zentrale Unterstützung für den Fortgang der Arbeit unentbehrlich war, worauf das Projektsekretariat wesentliche Aufgaben übernahm, die ursprünglich den Koordinierenden LeitautorInnen zugedacht waren, während das Organisationskomitee des ACRP-Projektes den Co-Chairs bei der Wahrnehmung ihrer Aufgaben behilflich war bzw. Aufgaben übernahm, die ursprünglich für letztere vorgesehen waren.

Beim Entwurf des ACRP-Projektes hatte man sich am internationalen IPCC Prozess orientiert, wobei wohl zu wenig berücksichtigt wurde, dass der internationale Expertenpool sehr viel größer als der nationale ist und der nationale Prozess daher viel stärker von der zeitlichen Verfügbarkeit und der Prioritätensetzung einzelner WissenschafterInnen abhängig ist. Auch zeitlich begrenzter Ausfall einzelner ForscherInnen hatte daher wesentliche Auswirkungen auf den Fortschritt und die Qualität des gesamten Projektes.

Das ACRP-Projekt „Austrian Panel on Climate Change Assessment Report“ umfasst auch die Analyse des Entstehungsprozesses des Österreichischen Sachstandsberichtes Klimawandel. Dazu wird ein eigener anonymisierter „Meta-Bericht“ als Unterstützung für die Konzeption möglicher zukünftiger nationaler Sachstandsberichte erstellt. 


\section{Mitwirkende}

Der AAR14 ist die gemeinsame Leistung zahlreicher Mitwirkender, die alle Wesentliches zu seinem Entstehen beigetragen haben. Es ist dem Organisationskomitee ein Anliegen, diese Leistungen zu würdigen:

\section{Autorinnen und Autoren}

Manche der rund 240 WissenschafterInnen haben einzelne Bände oder Kapitel koordiniert, andere haben einzelne Abschnitte, Absätze oder eine Graphik beigesteuert - ihrer aller Arbeit war für die Qualität des Berichtes entscheidend. Viele Koordinierende LeitautorInnen haben den Aufwand nicht gescheut, alle einschlägig in Österreich forschenden KollegInnen in die gemeinsame Arbeit einzubeziehen und einigen ist es gelungen, auch den Zeitplan einzuhalten. Manche haben kurzfristig während des Prozesses Verantwortung übernommen, um sichtbar gewordene Lücken zu schließen. Aus unterschiedlichen Gründen konnten nicht alle Koordinierenden LeitautorInnen bis zum Ende dabei bleiben - die meisten haben dennoch auf die eine oder andere Weise zum Gesamtwerk beigetragen.

\section{Qualitätskontrolle}

Keywan Riahi und Mathis Rogner, IIASA, haben in professioneller Weise den Begutachtungsprozess organisiert, und dafür über 70, weitgehend anonym bleibende, ehrenamtlich tätige ReviewerInnen zur Mitarbeit gewonnen. Durch über 2900 Kommentare und Fragen haben diese wesentlich zur Qualitätsverbesserung des Berichtes beigetragen. Die 13 Review-EditorInnen haben sichergestellt, dass den Kommentaren und Fragen der ReviewerInnen angemessen Rechnung getragen wird. Mit großer Ausdauer hat Mathis Rogner, IIASA, Rückmeldungen von allen Beteiligten eingefordert, Kommentar-und-Antworten-Tabellen erstellt, mehr als 17 Telefonkonferenztermine zustande gebracht, bei denen offene Punkte zwischen Review-EditorInnen, Koordinierenden LeitautorInnen und Organisationskomitee ausdiskutiert werden konnten, und auch sonst auf vielfältige Weise den Prozess vorangebracht.

\section{Projektmanagement}

Bei Laura Morawetz, BOKU, sind mit der Zeit alle Fäden zusammengelaufen. Fast unmerklich hat sie das Heft in die Hand genommen und die im Antrag bedauerlicherweise nicht vorgesehene Position der Projektmanagerin wahrgenommen. Trotz verwirrender Vielfalt an übermittelten Text- und Abbildungsversionen, Anfragen unterschiedlichster Art und immer wieder nicht eingehaltener Zeitpläne hat sie nie die Geduld und Übersicht verloren und vorausschauend auch das Organisationskomitee auf notwendige nächste Schritte hingewiesen. Ohne ihre unermüdliche und stets freundliche Ermutigung unterstützungsbedürftigen und selbst säumigen PartnerInnen gegenüber wäre dieser Bericht nicht fertig geworden. Wo immer Not an Mann oder Frau war, ist sie eingesprungen.

\section{Projektsekretariat}

Julian Matzenberger, TU Wien, hat die Verträge mit den Partnerinstitutionen aufgesetzt, die Homepage erstellt, wichtige Anleitungen für AutorInnen aus dem IPCC und GEA (Global Energy Assessment) Prozess an die AAR14 Bedürfnisse angepasst und bis Anfang 2014 die offiziellen Kontakte zur KPC gepflogen. Matthias Themeßl, CCCA, hat das Glossar erweitert und betreut, und die Literaturdatenbank aufgesetzt. Heidi Leitner, BOKU, hat sich um die Fertigstellung der Second Order Drafts verdient gemacht, und gemeinsam mit Iouli Andreev, Irene Schicker (BOKU) und Simon De Stercke (IIASA) die Literaturdatenbank befüllt, Kopien zitierter grauer Literatur gesammelt und gemeinsam mit Laura Morawetz für weitgehende Vereinheitlichung der Literaturverzeichnisse gesorgt. Die aufwändige Zusammenstellung der Originalgraphiken in mehreren Iterationen und das Einholen aller Copyrights oblag Benedikt Becsi (BOKU, TU Wien). Pat Wagner unterstützte von Seiten der IIASA Koordination und Kommunikation innerhalb des Organisationskomitees. Olivia Koland und Brigitte Wolkinger (Uni Graz) haben die Workshops und Review-Prozesse mit den Stakeholdern vorzüglich organisiert, den jeweiligen Informationsfluss sichergestellt und Kontakte 
weiter betreut, und die Second Order Draft-Versionen der Kapitel dafür mit aufbereitet. In den letzten Wochen ist Bano Mehdi (IIASA) zum Team dazu gestoßen und hat sehr effizient wichtige Lücken gefüllt, sei es die Übersetzung der Zusammenfassung für Entscheidungstragende, die Klärung offener Fragen zu Abbildungen oder die Erstellung von Vorlagen für die Titelei.

\section{Lektorlnnen}

Thomas Reithmayer, Matthias Litschauer, Thomas Gerersdorfer, Heidi Leitner und Huem Otero haben (unter teilweise auch knappen Zeitvorgaben) Manuskripte des ersten Entwurfes jedes Kapitels lektoriert. Mit bewundernswerter Flexibilität haben sie sich den - aufgrund stochastisch eingehender Manuskripte - stets wechselnden Zeitplänen angepasst. Thomas Reithmayer und Matthias Litschauer waren dann für das finale Lektorat der einzelnen Kapitel, der Synthese und der Zusammenfassung für Entscheidungstragende verantwortlich. Obwohl ursprünglich ganz andere Zeiträume für das finale Lektorieren vorgesehen waren, hat es vor allem Thomas Reithmayer ermöglicht, durch jonglieren seiner anderen Verpflichtungen Wartezeiten für die APCC Autorinnen auf ein Minimum zu reduzieren. Mathis Rogner hat, wo nötig, die englischen Übersetzungen von Kapitelüberschriften, Bildtexten und Kurzfassungen lektoriert und sprachlich überarbeitet.

\section{Layouterteam}

Das Layouterteam (Valerie Braun, Kati Heinrich, Tobias Töpfer) des Institutes für Interdisziplinäre Gebirgsforschung der Österreichischen Akademie der Wissenschaften unter der Leitung von Axel Borsdorf hat nicht nur sehr professionelle Layout-Arbeit unter teilweise knappen Zeitvorgaben geleistet, sondern ist dem Organisationskomitee auch hinsichtlich Verlag und Druck mit Rat und Tat zur Seite gestanden. Mit großer Selbstverständlichkeit haben die KollegInnen bis dahin übersehene Fehler ausgebessert, Abbildungen und Tabellen lesbarer gemacht, und eine zusätzliche Letztkontrolle des Gesamtwerkes vorgenommen, die sonst wohl unterblieben wäre. Die Erstellung eines ansprechenden Druckwerkes war ihnen sichtbar ein Anliegen. Auch als die fristgerechte Fertigstellung des Berichtes schon unmöglich erschien, arbeiteten sie unbeirrt weiter, reagierten freundlich auf immer neue Änderungswünsche seitens der APCC-Community und machten schließlich das Unmögliche doch möglich.

\section{Scientific Advisory Board}

Jill Jäger als SAB Vorsitzende hat in den ersten Phasen wichtigen und wertvollen Rat gegeben und den gesamten Prozess wohlwollend begleitet. Sie war ermutigend oder mahnend, jeweils zur richtigen Zeit und war ein sicherer Anker in dem bewegten Projektverlauf. Die beide weiteren Mitglieder des SAB, Daniela Jacob und Dirk Messner haben sich mit dankenswerter Geduld und äußert flexibel den sich ändernden Anforderungen angepasst und alle drei haben wertvolle Ratschläge für die Synthese und die Zusammenfassung für Entscheidungstragende gegeben. Jenen Anregungen, die nicht mehr im Werk selbst berücksichtigt werden konnten, wurde zumindest in der untenstehenden kritischen Reflexion Rechnung getragen, damit sie beim nächsten AAR berücksichtigt werden können.

\section{Projektkonzept}

Ganz am Beginn des Projektes stand Sebastian Helgenberger (BOKU), der bei der Erstellung des Antrages an den KLIEN/ACRP Wesentliches beigetragen und Kernelemente der Projektstruktur entwickelt hat. Er hat im Antrag auch bereits das Meta-Projekt angelegt, das es nun ermöglicht auch die (durchaus erwartbaren) Abweichungen zwischen der Theorie des Ablaufes, der sich am internationalen IPCC Prozess orientierte, und der Praxis auf nationaler Ebene zu dokumentieren und zu analysieren.

\section{Institutionelle Unterstützung}

Über 50 wissenschaftliche Einrichtungen ermöglichten ihren MitarbeiterInnen dankenswerterweise die ehrenamtliche Mitwirkung an der Erstellung und Qualitätssicherung des AAR14 und haben durch ihre wohlwollende Unterstützung entscheidend zum Sachstandsbericht beigetragen. 
Das Institut für Gebirgsforschung der Österreichischen Akademie der Wissenschaften ermöglichte großzügigerweise dem Layouterteam die volle Konzentration auf die Fertigstellung des Werkes und stellte seine beträchtliche Erfahrung bei der Herausgabe von Büchern zu Verfügung.

Besonders zu erwähnen ist die Unterstützung der Technischen Universität Wien, der Universität für Bodenkultur Wien, der Universität Graz und der IIASA, die es dem Organisationskomitee ermöglicht haben, wesentlich mehr Zeit in das Projekt zu investieren, als vorhergesehen. Diese vier Institutionen, in besonderem Ausmaße jedoch die Technische Universität Wien, die Universität für Bodenkultur und die IIASA haben darüber hinaus MitarbeiterInnen für das Sekretariat und die Qualitätskontrolle aus eigenen Mitteln finanziert, die Universität für Bodenkultur zusätzlich das Projektmanagement.

Der Klima- und Energiefonds (KLIEN) hat im Rahmen des Austrian Climate Research Programs das Projekt APCC und damit verbundene koordinierende Arbeit unterstützt. Die fachliche Arbeit und die koordinierende Tätigkeit der Wissenschaftler, einschließlich des Organisationskomitees, erfolgten ehrenamtlich. Dennoch, ohne die Unterstützung und den Rahmen des KLIEN/ ACRP wäre der AAR14 nicht entstanden. Der KLIEN hat sich auch bereit erklärt, gemeinsam mit dem Climate Change Centre Austria bei der Dissemination des Berichtes behilflich zu sein.

Die KPC als abwickelnde Stelle, insbesondere Biljana Spasojevic als Kontaktperson, war entgegenkommend bei Spezialwünschen, die mit dem etwas aus dem Rahmen der üblichen Forschungsprojekte fallenden Vorhaben einhergingen.

Der FWF hat innerhalb eines knapp bemessenen Zeitrahmens einen in finanzieller Hinsicht wichtigen Druckkostenbeitrag genehmigt, und darüber hinaus mit der dieser Bewilligung vorangehenden Prüfung den wissenschaftlichen Qualitätssicherungsprozeß bestätigt, dem das vorliegende Werk unterworfen war.

Der ÖAW Verlag erwies sich als sehr geduldig und hilfreich hinsichtlich unserer vielen Fragen, und war äußerst flexibel angesichts eines sich mehrfach verschiebenden Liefertermins für das Manuskript.

Das Climate Change Centre Austria (CCCA) wird, nach Abschluss des Projektes, die Produkte (AAR14, Literaturdatenbank, Glossar, etc.) in seine Obhut übernehmen, verfügbar halten und weiterführen. Das CCCA könnte, möglicherweise, der Träger des nächsten Österreichischen Sachstandsberichtes Klimawandel sein.

\section{Öffentliche Verwaltung}

Das Projekt war durchgängig mitgetragen durch die ideelle Unterstützung und die Bestärkung des Bewusstseins des Bedarfs der öffentlichen Verwaltung nach diesem Assessment Report. Allen voran ist die Sektion «Umwelt und Klimaschutz» des Bundesministeriums für Land-, Forstwirtschaft, Umwelt und Wasserwirtschaft zu nennen. Praktische Unterstützung erfuhr das Projekt auch durch die beiden, damals noch getrennten Bundesministerien für Wissenschaft und Forschung und für Wirtschaft, durch das Bundesministerium für Äußeres, das Bundesministerium für Verkehr, Infrastruktur und Technologie und das Bundeskanzleramt.

\section{PartnerInnen, Familien, Freundlnnen und KollegInnen}

Nicht zuletzt seien die Angehörigen, FreundInnen und nicht direkt beteiligten KollegInnen aller Mitwirkenden erwähnt, die zweifellos phasenweise unter deren zeitenger, teils sehr anspruchsvoller Beschäftigung mit dem AAR14 gelitten haben. Die Mitwirkung an IPCC Berichten soll Ehen gefährdet oder zerstört haben - das konnte beim APCC vermieden werden, aber die Geduld des Umfeldes vieler der Beteiligten - vor allem jener in den zentralen Positionen, denen in der letzten Phase Dauereinsatz abverlangt wurde - darf nicht unerwähnt bleiben.

\section{Ihnen allen sei an dieser Stelle seitens des Organisationskomitees sehr herzlich gedankt.}

\section{Qualitätssicherung}

In einem mehrstufigen Verfahren wurden zuerst Struktur und Inhalt des Gesamtwerkes erarbeitet, dann für jedes einzelne Kapitel genauer ausgearbeitet und die einzelnen Kapitel auf einander abgestimmt.

Für die Qualitätssicherung und die Organisation des Review-Prozesses zeichnete die IIASA verantwortlich. Jedes der 17 Kapitel wurde zweimal einem umfassenden Review unterzogen. 
Ein „First Order Draft“ (FOD) wurde einem ersten, teilweise internen, jedenfalls aber anonymen Review unterzogen. Die für den Qualitätssicherungsprozess zuständige IIASA sammelte die Kommentare und stellte diese in anonymer Form den Koordinierenden LeitautorInnen zur Verfügung. Letztere beantworteten die Kommentare und ließen diese und andere Verbesserungen und Erweiterungen in einen „Second Order Draft“ (SOD) einfließen.

Für jedes Kapitel in seiner Version als SOD wurden von der IIASA zwischen 3 und 6 internationale ReviewerInnen gewonnen, auch AutorInnen anderer Kapitel und TeilnehmerInnen des Stakeholderprozesses waren zusätzlich weiterhin zum Review eingeladen. Die anonymisierten Kommentare - zwischen 40 und 500 pro Kapitel - wurden gesammelt und wiederum den Koordinierenden LeitautorInnen zugestellt. In einer Tabelle wurde zu jedem Kommentar festgehalten, wie er abgearbeitet wurde - ob er umgesetzt, teilweise umgesetzt, oder abgelehnt wurde (in letzterem Fall mit Begründung warum).

Eine/ein Review EditorIn pro Kapitel überprüfte, ob die Kommentare und Anmerkungen zufriedenstellend behandelt wurden und erläuterte anschließend in einer oder mehreren Telefonkonferenzen den Koordinierenden LeitautorInnen seinen/ihren Befund. Co-Chairs und Mitglieder des Organisationskomitees nahmen ebenfalls an den Telefonkonferenzen teil, um Konsistenz in der Vorgangsweise zwischen den Kapiteln zu gewährleisten. In den meisten Fällen genügte eine Iteration und nachfolgende Ausarbeitung um die Freigabe des Kapitels durch die/den Review EditorIn zu erreichen. Die Tabellen mit den Kommentaren und Antworten sind einsehbar, um die nötige Transparenz zu gewährleisten.

Mit der schriftlichen Bestätigung der inhaltlichen Freigabe durch die Review-EditorInnen (sign-off-letter) konnte der Text ans Lektorat und anschleißend zum Layoutieren weitergeleitet werden. Nach jedem Schritt erfolgte eine Kontrolle durch die Koordinierenden LeitautorInnen, die letztlich die Verantwortung für ihr jeweiliges Kapitel tragen.

\section{Kritische Reflexion}

Selbstverständlich weist der aus unserer Sicht sehr gelungene Sachstandsbericht auch Schwächen auf. Einige davon sind uns schmerzlich bewusst. Dieser Bericht ist der erste Klimasachstandsbericht für Österreich, der einzige in der Intention vergleichbare Bericht wurde von der Akademie der Wissenschaften, Kommission Reinhaltung der Luft 1989-1992 erstellt. Bei jenem Bericht wurde von etwa einem Dutzend Fachleuten das aktuelle Wissen über den Klimawandel in Österreich zusammengetragen. Der AAR14 hatte demgegenüber neben der Erstellung eines Berichtes über einen zwischenzeitlich wesentlich umfangreicheren Sachstand auch das Ziel, die in Österreich auf dem Gebiet des Klimawandels, seiner Ursachen und Auswirkungen sowie zu den Anpassungs- und Mitigationsmaßnahmen Forschenden zusammenzuführen, um über verstärkten fachlichen Austausch Kapazität und Qualität der Klimaforschung in Österreich zu erhöhen. Deswegen wurde bei der Erstellung bewusst das Konzept des IPCC übernommen: Ein offener Prozess, bei dem erfahrene WissenschafterInnen als Verantwortliche für einzelne Kapitel sich bemühen, alle einschlägig Forschenden entweder direkt als AutorInnen, oder indirekt über ihre Publikationen in das Werk einzubeziehen.

Dieses zweite Ziel ist nicht in allen Bereichen oder nicht ausreichend gut gelungen. Es gibt Kapitel und Subkapitel, bei denen die Verantwortlichen die Chance zu nutzen wussten, gemeinsam an einer Publikation zu arbeiten und im Konsens der einschlägig Forschenden wurde das vorhandene Wissen innerhalb des begrenzten Platzes bestmöglich und umfassend dargestellt. Es gab aber - als anderes Extrem - auch Kapitel, in denen (zumindest zunächst) vorwiegend das eigene Werk bzw. jenes der eigenen Institution dargestellt wurde. In Einzelfällen wurden auch implizit einschränkende Grundansätze gewählt, die dazu führten, dass zahlreiche Arbeiten ausgeschlossen blieben, etwa dass in einem der Kapitel nur Arbeiten berücksichtigt werden, die ganz Österreich umfassen, nicht aber solche, die sich nur auf Teilregionen beziehen, oder in einem anderen Kapitel nur Arbeiten, die in das offizielle staatliche Berichtsschema fallen. In Einzelfällen mögen auch ausgeprägte persönliche Präferenzen eine Rolle gespielt haben. Im Zuge des mehrstufigen Reviewprozesses konnte in den meisten Fällen noch eine gewisse Öffnung bewirkt werden, doch konnte ein echtes, gemeinsames Assessment, bzw. die völlige Befreiung von den einschränkenden Voraussetzungen nicht mehr in allen Fällen erreicht werden. Wo es möglich war, wurde wenigstens versucht, solche Einschränkungen explizit zu machen. All jene Kolleginnen und Kollegen, deren Arbeiten solcherart nicht die gebührende Berücksichtigung fanden, bitten wir um Nachsicht. Wir laden Sie jedoch herzlich ein, uns auf diese Arbeiten aufmerksam zu machen, damit sie in die Datenbank des CCCA aufgenommen werden können und damit gegen dasselbe Versehen bei einer nächsten Auflage vorgebaut werden kann.

$\mathrm{Zu}$ Recht wurde von Gutachtern eingemahnt, dass Begriffe, Klassifizierungen, Gliederungen oder ähnliche gesamtheitliche Aspekte nicht immer systematisch durch alle Kapitel durchgezogen wurden. Dies ist auf die parallele Arbeit der verschiedenen 
AutorInnenteams zurückzuführen und die Schwierigkeit des Abgleiches, wenn nicht alle Kapitel zeitgerecht und damit zeitgleich vorliegen.

Im ursprünglichen Ablaufplan war reichlich Zeit vorgesehen um nach Vorliegen aller Kapitel, das Gesamtwerk auf Konsistenz, Vollständigkeit und Duplizierungen hin zu überprüfen. Leider zeigte sich, dass Termintreue auf der internationalen Eben offenbar leichter zu erreichen ist, als auf der nationalen. So wurden die AAR14 Publikationen zwar im Sinne des Auftraggebers fristgerecht fertig, doch fehlte es an Zeit für umfassende Quervergleiche. Für Hinweise auf Inkonsistenzen sind wir daher dankbar.

Nicht durch den Entstehungsprozess bestimmt ist das Problem, dass die Originalliteratur, auf der Band 2 und Band 3 beruhen, es oft an Klarheit bezüglich der Klimaszenarien fehlen läßt, für welche die jeweiligen Aussagen gelten. Hier wird künftig das in Errichtung befindliche Klimadatenzentrum des CCCA, das definierte Klimaszenarien für Impaktforschung zur Verfügung stellen wird, eine gewisse Abhilfe schaffen.

In zwei Bereichen konnten Gutachterwünsche meist nicht erfüllt werden: Der eine betrifft ökonomische Kostenabschätzungen für Schäden, Anpassungs- oder Minderungsmaßnahmen. Derartige Studien liegen bisher für Österreich nur sehr vereinzelt vor. An einer Abschätzung der Kosten des Nicht-Handelns wird derzeit gearbeitet - aufgrund der zeitgleichen Erstellung konnten die Ergebnisse nicht mehr in den vorliegenden AAR14 aufgenommen werden. Darüber hinaus zeigen aber auch diese Ergebnisse, dass es in vielen Bereichen noch an Daten fehlt.

Der zweite Themenblock betrifft Politik und Governance. Der AAR14 enthält wenig zum Thema politischer Rahmenbedingungen, Kompetenzverteilung und Entscheidungsfindung in der Klimapolitik, Österreichs Rolle in der EU- und internationalen Politik, oder ähnliche institutionelle Fragen. Dies geht einerseits auf ein tatsächliches Forschungsdefizit in diesem Bereich zurück, ist aber möglicherweise auch eine Folge der anfangs beschlossenen Gliederung des Sachstandsberichtes, welche diese Fragestellungen eher als Querschnittsmaterie verstanden wissen wollte, die dann jedoch nicht systematisch durch die Kapitel durchgezogen wurde - nicht zuletzt ein Hinweis auf noch fehlende Interdisziplinarität in der Klimaforschung.

Ein Problem systemischer Art betrifft die Aufnahme konkreter Fallbeispiele für Entwicklungen in Richtung gesellschaftlicher Transformation: In den meisten Fällen sind diese Pioniere - seien es Firmen, Gemeinden oder Regionen - bisher nicht Gegenstand wissenschaftlicher Untersuchungen. Sie entziehen sich damit weitgehend der Erfassung im AAR14, da weder exakte Beschreibungen der Vorhaben, noch Evaluierungen des bisherigen Erfolges vorliegen. Dies ist bedauerlich, denn gerade auf dieser Ebene geschieht in Österreich einiges, das zudem auch international interessant und jedenfalls der Analyse und Dokumentation wert wäre.

Es war ein Ziel des APCC Projektes, Forschungsdefizite zu erkennen, und damit eine Grundlage für einen österreichischen „Science Plan Klimawandel“ zu schaffen. Der in den letzten Abschnitten jedes Kapitels dargestellte Forschungsbedarf und die obigen Absätze zu den Caveats des AAR14 werden zum Kern des beim CCCA in Arbeit befindlichen Science Plans beitragen.

\section{Umgang mit Unsicherheit}

\section{Empirische Wissenschaft}

Erkenntnistheoretisch ist ein strenger Beweis für den anthropogenen Klimawandel nicht möglich, weil sich die Klimaforschung als eine empirische Wissenschaft versteht, die auf Beobachtung und Erfahrung gründet. Da die Theorie der Klimabeeinflussung durch den Menschen bereits seit über 30 Jahren der wissenschaftlichen Überprüfung unterworfen war, ist sie als wesentlich wahrscheinlicher einzuschätzen als andere vorgebrachte Hypothesen und es ist sachlich gerechtfertigt, sie weiteren Betrachtungen sowie auch Entscheidungen zugrunde zu legen.

Ein wesentlicher Teil der Analysen vergangener Änderungen und der Großteil der Zukunftsprojektionen beruhen auf Modellberechnungen. Es ist angebracht, Modelle kritisch zu betrachten: Sie geben die Natur bzw. die Wirklichkeit nie vollständig wieder, sie können immer nur Teilaspekte simulieren. Es ist daher wichtig, Modelle mit Überlegung einzusetzen, und nur dann, wenn sie für den spezifischen Zweck validiert sind. Andererseits sind Modelle die einzige Möglichkeit, quantitative Aussagen über komplexe Zusammenhänge zu ermitteln. Sie sind daher als Werkzeug unentbehrlich.

Mit dem Übergang von naturwissenschaftlichen zu gesellschaftspolitischen und ökonomischen Aspekten, d. h. mit dem Übergang von Band 1 und großteils Band 2 zu Band 3, gewinnen normative Vorgaben immer mehr an Bedeutung. Es wird versucht, diese jeweils explizit zu machen, doch mag dies nicht überall gelungen sein, da viele Vorgaben schon derart in der Denkkultur verankert sind, dass sie nicht mehr auffallen. 
All diesen Überlegungen zufolge ist es wichtig, Aussagen über den Klimawandel, seine Folgeerscheinungen und die Optionen für Maßnahmen durch die Angabe nachvollziehbarer Bewertungen zum Grad der (Un)sicherheiten der Aussagen zu ergänzen.

\section{Bewertung der Unsicherheit nach Muster des IPCC²}

Über das oben beschriebene grundsätzliche Problem empirischer Wissenschaften hinaus, können wissenschaftliche Aussagen aus verschiedenen Gründen unsicher sein. Unsicherheit entsteht zum einen durch einen Mangel an Information oder durch verschiedene Interpretationen sowohl dieser Informationen als auch darüber, was bekannt ist oder überhaupt bekannt sein kann. Unsicherheit kann viele Quellen haben, von bezifferbaren Fehlern in Daten bis hin zu mehrdeutig formulierten Konzepten und Terminologien oder unsicheren Projektionen über menschliches Verhalten. Unsicherheit kann deshalb entweder quantitativ angegeben werden, z.B. durch eine Auswahl von berechneten Werten aus verschiedenen Modellen, oder durch qualitative Aussagen, die das Urteil eines Expertenteams wiedergeben.

Der AAR14 ist um sorgfältige Kommunikation der Unsicherheiten bemüht - u. a. durch einen einheitlichen Umgang mit den Begrifflichkeiten, eine nachvollziehbare Darstellung und einheitliche Beschreibung und Evaluierung der wissenschaftlichen Beweise und ihrer Übereinstimmungen. Er folgt darin den Vorgaben des IPCC und orientiert sich hinsichtlich der deutschen Terminologie an der deutschsprachigen Übersetzung des IPCC Syntheseberichts $2007^{3}$. Der vom IPCC definierte Rahmen für den Umgang mit Unsicherheiten ist breit, da Material aus unterschiedlichen Disziplinen zu bewerten und eine Vielfalt an Ansätzen zu behandeln sind. Daten, Indikatoren und Analysen in den Naturwissenschaften sind im Allgemeinen von anderer Art als diejenigen, die bei der Bewertung von Technologieentwicklung oder in den Sozialwissenschaften herangezogen werden. Band 1 konzentriert sich auf erstere, Band 3 auf letztere, und Band 2 deckt Aspekte von beidem ab.

Zur Beschreibung von Unsicherheiten wird im gesamten Bericht ein einheitliches Konzept verwendet, das drei unterschiedliche Ansätze benutzt, von denen jeder eine bestimmte Sprache anwendet: Die Auswahl unter und innerhalb dieser drei Ansätze hängt sowohl vom Wesen der verfügbaren Daten ab als auch von der fachkundigen Beurteilung der Richtigkeit und Vollständigkeit des aktuellen wissenschaftlichen Verständnisses durch die AutorInnen.

Bei einer qualitativen Abschätzung wird Unsicherheit dadurch beschrieben, dass eine relative Einschätzung vermittelt wird für die Menge und Qualität an Beweisen (d.h. Informationen aus Theorie, Beobachtungen oder Modellen, die angeben, ob eine Annahme oder Behauptung wahr oder gültig ist) und für das Ausmaß an Übereinstimmung unter den Fachleuten (d.h. den Grad an Übereinstimmung in der Literatur zu einem bestimmten Ergebnis). Dies führt zu einer zweidimensionale Bestimmung der Unsicherheit. (Tabelle 1). Dieser Ansatz wird mit einer Reihe von selbsterklärenden Begriffen wie hohe Übereinstimmung, starke Beweislage; hohe Übereinstimmung, mittlere Beweislage; mittlere Übereinstimmung, mittlere Beweislage, usw. angewendet. Beide Dimensionen gemeinsam werden in ein Maß des „Vertrauens“ zusammengeführt (Tabelle 1, Skala ganz rechts).

Tabelle 1: Zweidimensionale Bestimmung des Vertravensbereiches - Verwendete Begriffe im AAR14

\begin{tabular}{|c|c|c|c|}
\hline \multirow{3}{*}{ 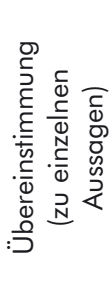 } & $\begin{array}{l}\text { Hohe Übereinstimmung } \\
\text { Schwache Beweislage }\end{array}$ & $\begin{array}{l}\text { Hohe Übereinstimmung } \\
\text { Mittlere Beweislage }\end{array}$ & $\begin{array}{l}\text { Hohe Übereinstimmung } \\
\text { Starke Beweislage }\end{array}$ \\
\hline & $\begin{array}{l}\text { Mittlere Übereinstimmung } \\
\text { Schwache Beweislage }\end{array}$ & $\begin{array}{l}\text { Mittlere Übereinstimmung } \\
\text { Mittlere Beweislage }\end{array}$ & $\begin{array}{l}\text { Mittlere Übereinstimmung } \\
\text { Starke Beweislage }\end{array}$ \\
\hline & $\begin{array}{l}\text { Niedrige Übereinstimmung } \\
\text { Schwache Beweislage }\end{array}$ & $\begin{array}{l}\text { Niedrige Übereinstimmung } \\
\text { Mittlere Beweislage }\end{array}$ & $\begin{array}{l}\text { Niedrige Übereinstimmung } \\
\text { Starke Beweislage }\end{array}$ \\
\hline
\end{tabular}

\footnotetext{
2 Dieser Abschnitt, wie auch die Kommunikation der Unsicherheit selbst, ist stark an die IPCC Guidance Notes angelehnt (Mastrandrea, M.D., C.B. Field, T.F. Stocker, O. Edenhofer, K.L. Ebi, D.J. Frame, H. Held, E. Kriegler, K.J. Mach, P.R. Matschoss, G.-K. Plattner, G.W. Yohe, and F.W. Zwiers, 2010: Guidance Note for Lead Authors of the IPCC Fifth Assessment Report on Consistent Treatment of Uncertainties. Intergovernmental Panel on Climate Change (IPCC). Available at <http://www.ipcc.ch>.)

3 Klimaänderung 2007, Synthesebericht. Zwischenstaatlicher Ausschuss für Klimaänderungen, Intergovernmental Panel on Climate Change IPCC, WMO/UNEP, 2008, ISBN 92-9169-122-4)
} 
Die Vertrauensskala wird als quantitative Einschätzung von Unsicherheit mittels fachkundiger Beurteilung der Richtigkeit zugrundeliegender Daten, Modelle oder Analysen verstanden. Es wird die in Tabelle 2 beschriebene Skala angewandt, um die geschätzte Wahrscheinlichkeit für die Richtigkeit eines Ergebnisses auszudrücken.

Tabelle 2: Beschreibung der Richtigkeit - Verwendete Begriffe im AAR14

\begin{tabular}{|ll|}
\hline Terminologie & Vertrauensbereich (Grad des Vertrauens bezüglich der Richtigkeit) \\
\hline sehr hohes Vertrauen & in mindestens 9 von 10 Fällen korrekt \\
\hline hohes Vertraven & in etwa 8 von 10 Fällen korrekt \\
\hline mittleres Vertraven & in etwa 5 von 10 Fällen korrekt \\
\hline geringes Vertraven & in etwa 2 von 10 Fällen korrekt \\
\hline sehr geringes Vertrauen & in weniger als 1 von 10 Fällen korrekt \\
\hline
\end{tabular}

Ist hingegen eine vollumfänglich quantitative Bewertung möglich (d.h. liegen so viele Daten bzw. Modellergebnisse vor, dass auch die Wahrscheinlichkeit des Eintreffens einer bestimmten Aussage quantitativ angegeben werden kann), so werden die in Tabelle 3 angegebenen Wahrscheinlichkeitsbereiche verwendet, um die geschätzte Eintrittswahrscheinlichkeit auszudrücken. Die Wahrscheinlichkeit bezieht sich auf die Bewertung der Wahrscheinlichkeit eines gut definierten Ergebnisses, das eingetreten ist oder zukünftig eintreten wird. Sie kann aus quantitativen Analysen oder Expertenmeinungen abgeleitet werden.

Tabelle 3: Wahrscheinlichkeiten - Verwendete Begriffe im AAR14

\begin{tabular}{|ll|}
\hline Terminologie & Wahrscheinlichkeit des Eintretens des Ereignisses bzw. der Auswirkung \\
\hline praktisch sicher & $>99 \%$ Wahrscheinlichkeit \\
\hline sehr wahrscheinlich & $>90 \%$ Wahrscheinlichkeit \\
\hline wahrscheinlich & $>66 \%$ Wahrscheinlichkeit \\
\hline wahrscheinlicher als nicht & $>50 \%$ Wahrscheinlichkeit \\
\hline etwa so wahrscheinlich wie nicht & $33 \%-66 \%$ Wahrscheinlichkeit \\
\hline unwahrscheinlich & $<33 \%$ Wahrscheinlichkeit \\
\hline sehr unwahrscheinlich & $<10 \%$ Wahrscheinlichkeit \\
\hline außergewöhnlich unwahrscheinlich & $<1 \%$ Wahrscheinlichkeit \\
\hline
\end{tabular}

In Zusammenfassungen und Kernaussagen kann die Evaluierung und Beschreibung der Unsicherheiten auch vereinfachend zusammengefasst werden:

- Für Forschungsergebnisse (bzw. Kernaussagen) mit hoher Übereinstimmung zu einer einzelnen Aussage UND starker Beweislage die Anzahl und Qualität unabhängiger Quellen betreffend wird in der Regel der Vertrauensbereich (,sehr hohes Vertrauen“, Tabelle 2) angegeben.

- Für Ergebnisse mit hoher Übereinstimmung ODER starker Beweislage, wenn also nicht beides vorhanden ist, wird entweder der Vertrauensbereich oder eine Kombination der Bewertungen, also z. B. „starke Beweislage, mittlere Übereinstimmung”, gemäß Tabelle 1 angewendet.

- Für Forschungsergebnisse (bzw. Kernaussagen), für die ausreichend Daten und Modellergebnisse vorliegen, sodass eine quantitative Wahrscheinlichkeit für das Zutreffen der Aussage angegeben werden kann, erfolgen Wahrscheinlichkeitsaussagen wie „sehr unwahrscheinlich“, „sehr wahrscheinlich“ oder „praktisch sicher“.

Trotz dieser recht ausführlichen Vorgaben zur Beschreibung von Unsicherheiten, bleibt in der praktischen Handhabung Spielraum. Die Bewertungen in AAR14 sind daher - wie auch im IPCC Bericht - vermutlich nicht vollständig, aber doch weitgehend konsistent. 
Wir freuen uns, das vorliegende Werk der Öffentlichkeit übergeben zu können. Diese umfassende Zusammenstellung von Forschungsergebnissen zum Klimawandel in Österreich, seinen Auswirkungen und relevanten Minderungs- und Anpassungsmaßnahmen möge für Entscheidungstragende in der Politik und Verwaltung, in Kommunen und in der Wirtschaft zu einer wertvollen Hilfe werden. Darüber hinaus hoffen wir damit einen Beitrag zum besseren Verständnis der Herausforderung Klimawandel zu leisten und so der österreichischen Klimapolitik neuen Aufschwung zu geben.

Das Organisationskomitee:

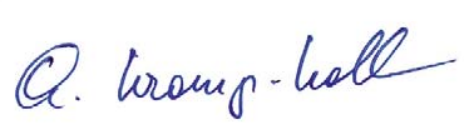

Helga Kromp-Kolb

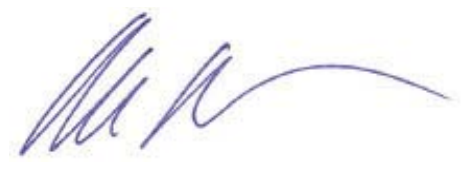

Nebojsa Nakicenovic

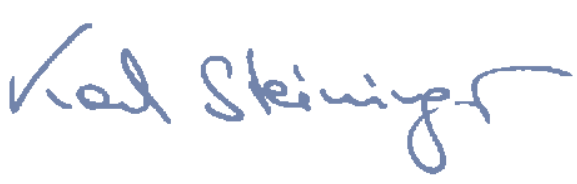

Karl Steininger 
